\title{
An Optimized Particle Swarm Optimization (PSO) Algorithm Smoothing the Output of Wind Power
}

\author{
X.Y. Zhang, N. Zhu, M.L. Yuan, J.Y. Xu, H.T. Dai \\ Guodian United Power Technology Company LTD \\ Beijing, China
}

\begin{abstract}
The output power of wind turbine caused by the variation of wind fluctuates badly, which causes instability of the Power Grid. To smooth the output of wind power to Grid, an Energy Storage System (ESS) solves this problem by absorbing and releasing the wind turbine energy. The smooth performance depends on the State of Charge (SOC) of the ESS. Considering the SOC of the ESS, an optimized Particle Swarm Optimization (PSO) algorithm is designed in this paper, where the SOC of the ESS is the boundary condition. A penalty mechanism is designed to ensure the wind power system's feasibility. The wind power system with ESS based on MATLAB is modelled. The optimized PSO algorithm is used to control output wind power. The simulation results verified it.
\end{abstract}

Keywords-wind power; smoothing; Energy Storage System(ESS); PSO algorithm

\section{INTRODUCTION}

As the proportion of wind power in Grid increases, the fluctuated wind power takes bad influences to Grid, such as the peak adjustment problem, frequency variation, operation instability and so on[1][2], It's very necessary to smooth the wind power by installing Energy Storage System (ESS). There are lots of researches on the application of ESS in wind power The ESS installed in the wind generation system can improve the dynamic response characteristics caused by variation, to make the wind generation system friendlier to Grid[3][4]

Various algorithms about controller of ESS installed in wind power system were proposed. In [5], it designed a Firstorder low pass filter to smooth the wind power fluctuation. Besides this, an average smooth scheme is very popular [6-8]. In paper [6], it set the averaged wind speed as a current reference; In [7] and [8], the difference value between the average value and standard deviation of wind speed as the references; In [9], it takes half the difference value above as a reference. Though these schemes can smooth the output power to some extent, they didn't take the SOC of ESS into consideration and the smooth properties are not optimum.

In [10] [11], it takes the SOC of ESS as the constraint condition, while the ESS and wind output power are not optimum.

In this paper an optimized Particle Swarm Optimization (PSO) algorithm is proposed for direct-drive wind turbine. It reaches an optimal smooth performance and the SOC of ESS is under range. The simulation results verified this scheme.

\section{ANALYSIS ON WIND TURBINE WITH ENERGY STORAGE SYSTEM}

A. Power Flow from Direct-Drive Wind Turbine to Grid

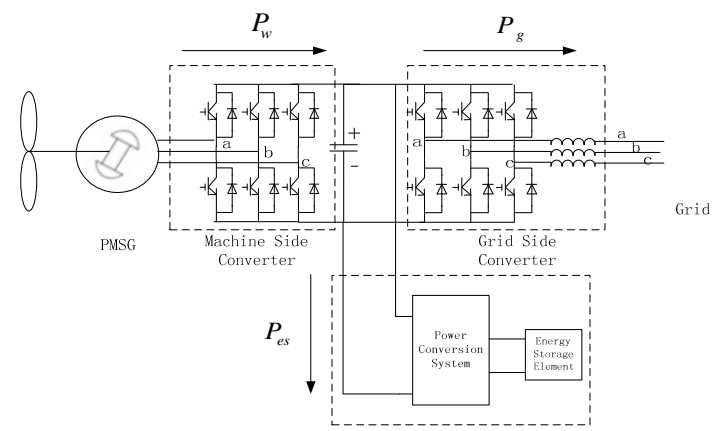

FIGURE I. TOPOLOGY OF DIRECT-DRIVE WIND TURBINE WITH ENERGY STORAGE SYSTEM

In Figure 1, it's a Direct-Drive Wind Turbine, and an energy storage element is paralleled to the DC bus , through the power conversion system, it absorb or release the power generated by wind turbine to smooth the output wind power.

Based on the energy conservation law,

$$
P_{w}=P_{g}+P_{e s}
$$

In(1), $P_{w}$ represents the active power converted from the mechanical energy of wind turbine, $P_{g}$ represents the output power to Grid, $P_{\text {es }}$ represents the energy absorbed by energy storage system. The goal is obtaining a smooth $P_{g}$ curve.

The forward direction is shown as in the figure $1, P_{e s}>0$ means that the Energy Storage System, for short ESS, is absorbing energy, $P_{e s}<0$ means the ESS is releasing energy. $P_{g}$ is the control objective, the smoothness of $P_{g}$ curve represents the advantage of the smooth scheme.

\section{B. Analysis on State of Charge of Energy Storage System}

It's assumed that the number of the sampling points is $N$, the time interval between sampling points is a constant, which 
expressed as $T$. Then the power formula at step $i$ is as shown in (2).

$$
P_{w}(\mathrm{iT})=P_{g}(\mathrm{iT})+P_{e s}(\mathrm{iT})
$$

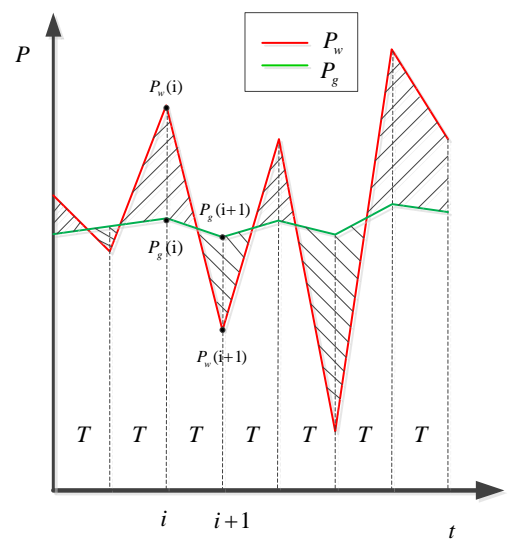

FIGURE II. POWER CURVES OF $P_{w} P_{g}$

As shown in Fig .2, we assume that the red curve is $P_{w}$, and the green curve is $P_{g}$.During the operation, ESS always absorbs or release the energy. If the target $P_{w}$ is above $P_{g}$, ESS stores energy; once below $P_{g}$, ESS releases energy. No matter how the process, ESS can't operate out of range.

Based on this, the smooth scheme has to take the SOC of ESS into consideration. It's a constraint requirement.

The initial storage range is set as $\left[e_{\min }(0), e_{\max }(0)\right]$ $\left(e_{\min }(0)<0, e_{\max }(0)>0\right.$ ). To make sure ESS working in normal range, the following relationship $e_{\min }(i T) \leq 0$ and $e_{\max }(i T) \geq 0$, are essential requirements.

In every interval period, the power energy stored in ESS can be expressed in the following equation:

$$
\begin{aligned}
& e(\mathrm{i})=\int_{i T}\left[f\left(\left(P_{w}(\mathrm{i})\right)-f\left(P_{g}(\mathrm{i})\right)\right] \mathrm{dt}\right. \\
& \left\{\begin{array}{l}
e_{\max }(\mathrm{i}+1)=e_{\text {max }}(\mathrm{i})-e(\mathrm{i}) \\
e_{\text {min }}(\mathrm{i}+1)=e_{\text {min }}(\mathrm{i})-e(\mathrm{i})
\end{array}\right.
\end{aligned}
$$

During the whole process, the smooth scheme must make sure $e_{\max }>0$ and $e_{\min }<0$.

\section{PARTICLE SWARM Optimization (PSO) ALGORITHM MODEL}

PSO algorithm is a kind of heuristic search technology. It adopts the idea of information sharing among biotic community, and seeks an optimum solution in a complex space by assistance among individuals. PSO algorithm simulates a social intercourse of swarm, at the beginning; it generates a initial population and a initial speed for every particle randomly. In the flying process, every particle can adjust its speed and locus by itself and its companion's flying experience, the whole swarm can fly to a better region.

It's assumed that in a D dimension space, PSO algorithm initials $n$ numbers of random particles, the position of every particle is a potential solution. In every iteration, the particle updates itself through tracking two variables, the first one is an optimal solution calculated by itself so far, which is called individual extremum $p_{\text {best }}$, it presents the particle's best flying experience itself; the second one is a optimal solution for the whole swarm so far, which is called global extremum $g_{\text {best }}$, it presents the swarm's flying experience[12].

The position vector of the i-th particle represents as following:

$$
X_{i}=\left\{x_{i 1}, x_{i 2}, \cdots, x_{i D}\right\}, i=1,2, \cdots, n .
$$

The flying speed vector of the i-th particle represents as following:

$$
V_{i}=\left\{v_{i 1}, v_{i 2}, \cdots, v_{i D}\right\}, i=1,2, \cdots, n .
$$

The best position vector of the i-th particle represents as following:

$$
P_{i}=\left\{p_{\text {besti1 }}, p_{\text {besti2 }}, \cdots, p_{\text {bestiD }}\right\}, i=1,2, \cdots, n .
$$

The best position vector of the whole swarm represents as following:

$$
G_{i}=\left\{g_{\text {besti1 }}, g_{\text {besti2 }}, \cdots, g_{\text {bestiD }}\right\}, i=1,2, \cdots, n \text {. }
$$

The particle updates its own speed and position as followed:

$$
\begin{gathered}
V_{i}^{t+1}=V_{i}^{t}+c_{1} r_{1}\left(P_{i}-X_{i}^{t}\right)+c_{2} r_{2}\left(G_{i}-X_{i}^{t}\right) \\
X_{i}^{t+1}=V_{i}^{t+1}+X_{i}^{t}
\end{gathered}
$$

In formula (5), $c_{1}=1, c_{2}=2, c_{2}$ is the learning factor, it's a constant. Its physical significance is the pulling power to adjust its own position and global optimal position, $r_{1}$ and $r_{2}$ are random number between 0 and 1.The first part of the expression represents the pre-position of the particle ; the second part represents "cognitive factor", it's the "thought" of the particle itself; the third part represents "social factor", it represents the information sharing and corporation.

As it is referred in Part 2.2.We must keep the SOC of ESS in a feasible range. In this algorithm, a penalty mechanism is planted into it. 
The position of the selected particle will be calculated through equation (3) and (4).It represents the state of ESS. If it doesn't meet the constraint requirement $e_{\max }>0$ and $e_{\min }<0$, then a penalty function will be added into the properties function. So this over ranged particle will be deleted before the next iteration.

The performance index criteria of $P_{g}$ is shown in (7). It's expressed as $J(J>0)$. The value lower, the performance better.

$$
J=\sum_{i=2}^{N} \sum_{j=1}^{\mathrm{N}-1}\left[P_{g}(i)-P_{g}(j)\right]^{2}
$$

The flow chart of PSO algorithm is as followed in Fig 3:

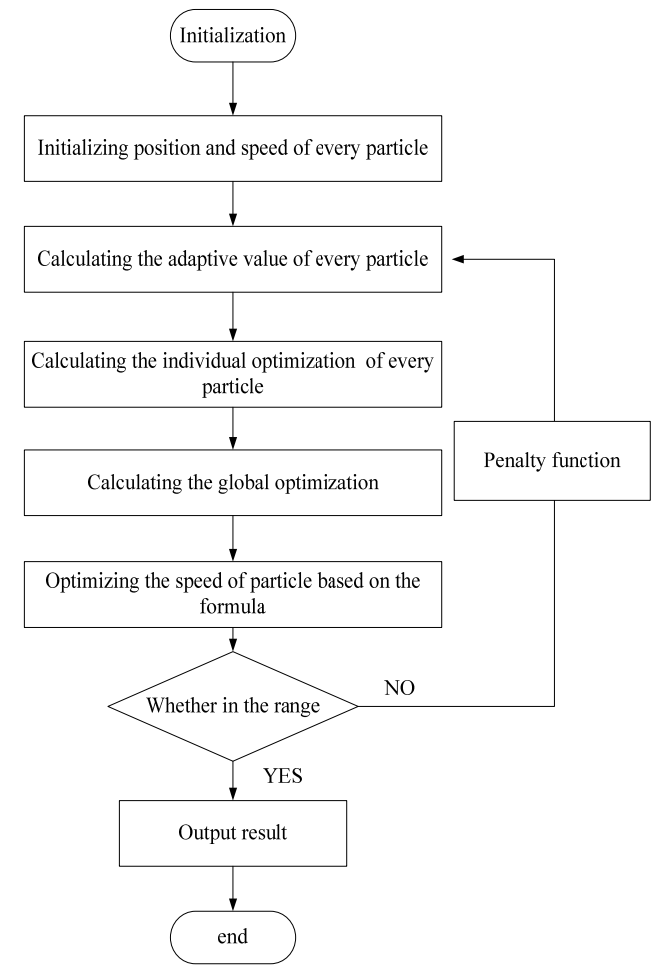

FIGURE III. FLOW CHART OF OPTIMIZED PSO ALGORITHM

\section{SimUlATION MODEL AND RESUlTS ANALYSIS}

\section{A. Simulation Model Parameters}

In the PSO algorithm, the learning factors are set as $c_{1}=1, c_{2}=2$, it generates random particles 80 times; every time the number of particles is 300 , the iteration is set as 1000 times every process. And the generation times and the iteration times can be set larger ones, which will result in a better value, while the larger, the time longer.

In this paper, the data is from the real output power of a $1.5 \mathrm{MW}$ wind turbine, on the base of the assumption of the wind prediction is accurate, we adopt the PSO algorithm, and the sample interval is set as 15 minutes, namely,. The storage range of ESS is $[-1.5 \mathrm{KJ},+1.5 \mathrm{KJ}]$.

$$
\left\{\begin{array}{l}
T=900 \mathrm{~s} \\
e_{\max }=1500 \mathrm{~J} \\
e_{\min }=-1500 \mathrm{~J}
\end{array}\right.
$$

Based on the predicted wind data, we set a limit value for the particles' speed and position. In this algorithm, a penalty function was set; it's that if the position of the particle is beyond the storage range, then a rather big value was added on the adaptive value, so this particle will be eliminated in the calculation.

\section{B. Simulation Results}

The best particle's global optimization adaptive value among the particle swarm during the iterations is as the following figure 4:

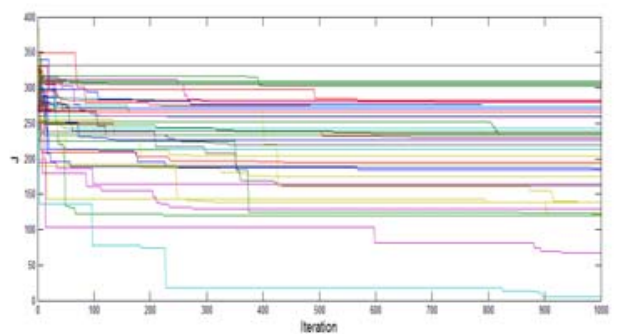

\section{FIGURE IV. PERFORMANCE OF ALL THE PARTICLES DURING ITERATIONS}

In figure 4, there are various curves. They are the iteration processes of the particle swarm. In the simulation, we set the number of particles 80 . We can see though different, the performance index $J$ of them all intend to smaller values. It verifies that this algorithm can seek to an optimum solution during iteration.

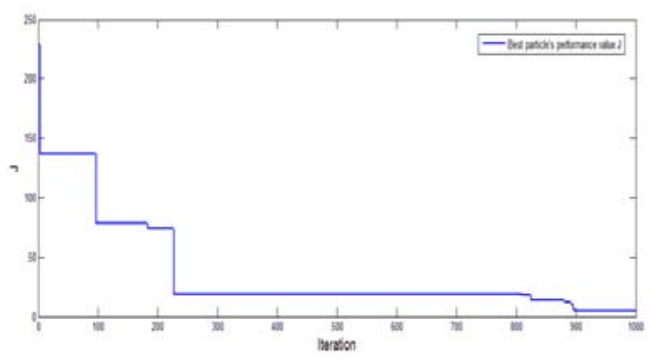

FIGURE V. PERFORMANCE OF THE BEST PARTICLE DURING ITERATIONS

We choose the best particle, whose performance is optimal among the particle swarm. As shown in figure 5. It reaches a best $J$ in the end among all the paths. 


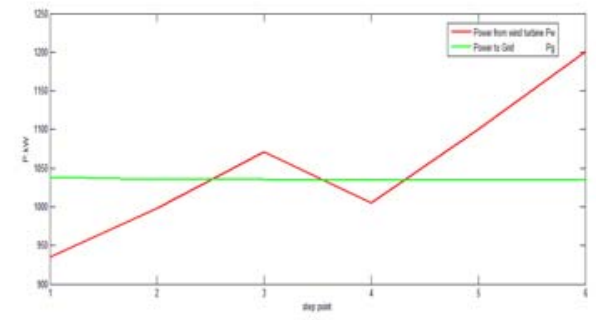

FIGURE VI. OUTPUT POWER OF WIND TURBINE TO GRID

From figure 4, the adaptive value decreases, it verified that the PSO algorithm can approach to the optimal solution. From figure 6, the output power $P_{g}$ is smoother than $P_{w}$ to a great extent. It verified the PSO algorithm is a superior scheme in power smoothing.

\section{CONCLUSION}

In this paper, the algorithm smooth the direct-drive output power of wind turbine, an optimized Particle Swarm Optimization (PSO) algorithm was designed. It sets a penalty mechanism to the over ranged solution, so all the unreasonable results are kept out. On one side, the ESS running in the working range, on the other side, the output power of wind turbine reached optimal. The simulation results verified it.

Besides, this algorithm can be used in any fluctuated power plant. However, there are still some aspects need to be pay attention to. This algorithm depends on the accuracy of the wind prediction; above this, a long-time optimization of wind power need to be researched on.

\section{REFERENCES}

[1] Liu Shinian, SU Wei, WEI Zengfu. Forecast of Energy Storage Applied in Wind Power Integration. Science and Technology Management Research, (2).120-122,2014.

[2] [GU Junhe, LIU Jianping, JIANG Hao. Literature Review on the Influence of Wind Power on System Frequency and Frequency Regulation Technologies of Wind Power. Modern Electric Power,(2).46-51,2015(1).

[3] YUAN Xiaoming, CHENG Shijie, WEN Jinyu. Prospect Analysis of Energy Storage System in solving large scaled Wind Power Generation. Automation of Electric Power System, (2), 14-18,2013(1).

[4] [4] Aghatehrani, R., Kavasseri, R., Thapa, R.C., Power and Energy Society General Meeting, (5), 1 - 6,2010.

[5] [5] Yu Hang, Simulation Research on Smoothing the Wind Power Fluctuation by Using Energy Storage System. Northwest Dianli University,(4),2010.

[6] Wu Jiao. Active Power Smoothing Control of Wind Generation. System. LanZhou University of Technology, (4),2011.

[7] LIAO Yong, HE Jin-bo, YAO Jun, ZHUANG Kai. Power Smoothing Control Strategy of Direct-driven Permanent Magnet Synchronous, Generator for Wind Turbine With Pitch Angle Control and Torque Dynamic Control. Proceedings of the CSEE,(5),71-77,2009(18).

[8] Hu Xuesong. Study on Power Smoothing Control Strategy and Control System Design of Direct-driven Permanent Magnet Synchronous Generator for Wind Turbine. Chongqing University,(4),2010.

[9] Xiong Qian, Liao Yong. Power Smoothing Control Strategy for Directdriven Wind Power System with Flywheel Energy Storage System. East China Electric Power,(5)1533-1539, 2012,40( 9).

[10] CHEN Yueyan, LI Xiangjun, HAN Xiaojuan, LIANG Tingting, Hui Dong. Control Strategy of Smoothing Wind Power Output Using Battery Energy Storage Based on Moving Average Method and Wind
Power Volatility Rate Constraint. Electric Power Construction,(5)1$5,2014,34(7)$.

[11] DING Ming, WU Jianfeng, Zhu Chengzhi, ZHAO Bo, CHEN Zinian, Luo Yaqiao. A Real-time Smoothing Control Strategy With SOC Adjustment Function of Storage Systems. Proceedings of the CSEE,(5),22-29,2013,33(1).

[12] CHEN Min-You, ZHANG Cong-Yu, LUO Ci-yong. Adaptive Evolutionary Particle Swarm Algorithm for Multi-Objective Optimization. Journal of System Simulation,(2), 7061$7065,2009,21(22)$. 